\title{
HEAT CONDUCTION IN A MELTING SOLID*
}

\author{
BY \\ H. G. LANDAU** \\ Ballistic Research Laboratories, Aberdeen Proving Ground
}

1. Introduction. There are a number of problems in heat conduction in which one material is transformed into another with generation or absorption of heat. Examples are the melting or freezing of a solid and the progress of a temperature-dependent chemical reaction through a solid. Some of these problems have been treated under various assumptions. ${ }^{1}$ The problem considered here is the melting of a solid when the liquid is removed immediately on formation. This problem seems to have been considered before only by Soodak, ${ }^{2}$ who gave the steady state rate of melting and carried out numerical integration by finite differences for some special values.

We first state a general problem and a few results. Then the problem is specialized to make possible. a more detailed treatment. For the case of a semi-infinite melting ${ }^{3}$ solid with its face heated at a constant rate, the exact steady state solution is derived, and the problem is put in a form involving only a single parameter. Numerical integrations have been carried out for this case for several values of the parameter extending over the entire possible range of the physical constants involved.

2. Statement of problem. We consider one-dimensional heat conduction in a solid which initially extends from $x=0$ to $x=a$. The face $x=a$ is insulated, while heat flows in through the other face at a rate $H(t)$ per unit area. If heating continues long enough, the face at $x=0$ reaches the melting temperature $T_{m}$ and melting commences. The liquid is removed immediately on formation, say by being blown away, so that the face of the solid, which was initially at $x=0$, moves inward and at time $t$ is at the position $x=s(t)$. If the heating rate decreases enough, the face temperature may drop below $T_{m}$ so melting stops temporarily and $s(t)$ remains constant; then melting may resume if $H(t)$ increases sufficiently. The temperature of the solid $T(x, t)$ and the thickness melted $s(t)$ are the quantities we wish to find.

In addition to the terms defined above we use the following:

$c=$ specific heat, $\rho=$ density, $k=$ thermal conductivity, $x=$ distance from initial position of heated face, $T_{0}(x)=$ initial temperature distribution, $L=$ latent heat of fusion.

Then the equations describing the process will be as follows:

$$
c \rho \frac{\partial T}{\partial t}=\frac{\partial}{\partial x}\left(k \frac{\partial T}{\partial x}\right), \quad s(t)<x<a, \quad t>0
$$

${ }^{*}$ Received Nov. 19, 1948.

**Now with the Committee on Mathematical Biology, University of Chicago.

${ }^{1}$ Several references are given in H. S. Carslaw and J. C. Jaeger, Conduction of heat in solids, Oxford, 1947, p. 71. An additional reference is L. I. Rubinstein, Isvestia Akad. Nauk SSSR, Sér. Géograph. Géophys. 11, 37-54 and 489-496 (1947).

${ }^{2} \mathrm{H}$. Soodak, Effects of heat transfer between gases and solids, Ph.D. thesis, Duke Univ., Durham, N. C. (1943).

"Although the term "melting" is used, the treatment in this paper would apply if the phase change were directly from solid to gas by sublimation. 


$$
\begin{gathered}
T(x, 0)=T_{0}(x) \leq T_{m}, \quad 0 \leq x \leq a, \quad t=0, \\
\frac{\partial T}{\partial x}=0, \quad x=a, \quad t>0, \\
H(t)=-k \frac{\partial T}{\partial x}+\rho L \frac{d s}{d t}, \quad x=s(t), \quad t>0 .
\end{gathered}
$$

The last equation expresses the fact that the heat input $H(t)$ equals the rate of heat flow into the solid plus the rate of heat absorption by melting. Condition (2.4) can be seen to hold both during melting and non-melting if we specify that at the heated face $x=s(t)$

$$
\begin{aligned}
& \frac{d s}{d t} \geq 0, \quad \text { for } T(s(t), t)=T_{m}, \\
& \frac{d s}{d t}=0, \quad \text { for } T(s(t), t)<T_{m} .
\end{aligned}
$$

The essential difficulty in the problem is in the determination of the unknown moving boundary $s(t)$. The problem can be seen to be non-linear because two different heat input functions, $H_{1}(t)$ and $H_{2}(t)$, would give different boundaries, $s_{1}(t)$ and $s_{2}(t)$, so that the two solutions would not apply in the same region in the $x, t$ plane, and the solution for the sum $H_{1}(t)+H_{2}(t)$ cannot be obtained by addition. In this paper the existence and uniqueness of $T(x, t)$ and $s(t)$ are assumed.

The moving boundary can be eliminated by the transformation $\xi=(a-x) /[a-s(t)]$. Then the boundaries are fixed at $\xi=0$ and $\xi=1$. Equations (2.1) to (2.6) become

$$
\begin{gathered}
c \rho\left[\frac{\partial T}{\partial t}+\frac{\xi}{a-s} \frac{d s}{d t} \frac{\partial T}{\partial \xi}\right]=\frac{1}{(a-s)^{2}} \frac{\partial}{\partial \xi}\left(k \frac{\partial T}{\partial \xi}\right), \quad 0<\xi<1, \quad t>0, \\
T(x, 0)=T_{0}(\xi)<T_{m}, \quad 0 \leq \xi \leq 1, \quad t=0, \\
\frac{\partial T}{\partial \xi}=0, \quad \xi=0, \quad t>0, \\
H(t)=\frac{k}{a-s} \frac{\partial T}{\partial \xi}+\rho L \frac{d s}{d t}, \\
\frac{d s}{d t} \geq 0, \quad \text { for } T(1, t)=T_{m}, \xi \xi=1, \quad t>0 . \\
\frac{d s}{d t}=0, \quad \text { for } T(1, t)<T_{m},
\end{gathered}
$$

With the equations in this form the non-linearity of the problem can be seen from the fact that $s$ and its derivative, which occur in the coefficients of the differential equation, depend on $\partial T / \partial \xi$ at $\xi=1$. We do not use the equations in the above form but they might be useful for numerical integration of the general problem. 
Gauss's theorem applied to the heat conduction equation (2.1) gives

$$
0=\iint_{D}\left[\frac{\partial}{\partial x}\left(k \frac{\partial T}{\partial x}\right)-c \rho \frac{\partial T}{\partial t}\right] d x d t=\int_{C}\left[k \frac{\partial T}{\partial x} d t+c \rho T d x\right]
$$

where $D$ is any region in the $x, t$-plane to which the theorem applies, and $C$ is its boundary. Applying (2.7) to the region bounded by the lines $t=0, t=t_{1}, x=a$, and the curve $x=s(t)$, we obtain for its two terms:

$$
\begin{gathered}
\int_{C} k \frac{\partial T}{\partial x} d t=\int_{0}^{t_{1}}\left[H(t)-\rho L \frac{d s}{d t}\right] d t=\int_{0}^{t_{1}} H(t) d t-\rho L s\left(t_{1}\right), \\
\int_{C} c \rho T d x=\int_{0}^{a} c \rho T_{0}(x) d x-\int_{s\left(t_{1}\right)}^{a} c \rho T\left(x, t_{1}\right) d x-\int_{0}^{t_{2}}\left[c \rho T(s(t), t) \frac{d s}{d t}\right] d t .
\end{gathered}
$$

In the last integral $d s / d t=0$ except when $T(s(t), t)=T_{m}$; this integral, therefore, equals $c \rho T_{m} s\left(t_{1}\right)$, and we have

$$
\begin{aligned}
\int_{0}^{t} H(t) d t=\rho \int_{o(t)}^{a} & {\left[c T(x, t)-c T_{0}(x)\right] d x } \\
& +\rho\left[\left(L+c T_{m}\right) s(t)-\int_{0}^{s(t)} c T_{0}(x) d x\right],
\end{aligned}
$$

where we have dropped the subscript 1 from $t_{1}$. This equation, as well as (2.7), holds when $k$ and $c$ are functions of $T$. It can be seen that (2.8) equates the total heat inflow, up to time $t$, to the change in heat content of the remaining solid plus that of the liquid.

Since $T(x, t) \leq T_{m}$, the first term on the right of (2.8) is bounded. Thus if $\int_{0}^{t} H(t) d t$ increases to large enough values, then $s(t)$ must finally reach its largest possible value, $s(t)=a$; that is, if heating continues long enough the entire solid melts. The time, $t_{e}$, when this occurs will be given by

$$
\int_{0}^{t_{\bullet}} H(t) d t=a \rho\left[L+c T_{m}-a^{-1} \int_{0}^{a} c T_{0}(x) d x\right] .
$$

The rate of melting at this time can be obtained from (2.3), (2.4), and the continuity of $\partial T / \partial x$. The result is that at the time $t_{0}$ when melting is completed,

$$
\frac{d s\left(t_{s}\right)}{d t}=\frac{H\left(t_{e}\right)}{\rho L} .
$$

During melting, $\partial T / \partial x \leq 0$ at $x=s(t)$, otherwise $T(x, t)>T_{m}$ for some $x$. Consequently from (2.4), $d s / d t \leq H(t) / \rho L$. Hence if $H(t)$ is non-decreasing, the melting rate will reach a maximum at the time $t_{\mathrm{e}}$.

The value of $T(x, t)$ during any period when there is no melting is not difficult to find, under the assumption that $c$ and $k$ are constant. This value can be found by various well-known methods such as the use of the Laplace transformation or the method of sources and sinks. ${ }^{4}$ The result is that before melting starts

‘Carslaw and Jaeger, loc. cit., ch. X. 


$$
\begin{aligned}
T(x, t)=\left(4 b^{2} \pi t\right)^{-1 / 2} & \int_{0}^{a} T_{0}(\xi) \sum_{n=-\infty}^{\infty}\left\{\exp \left[-\frac{(x-\xi+2 n a)^{2}}{4 b^{2} t}\right]\right. \\
& \left.+\exp \left[-\frac{(x+\xi-2(n+1) a)^{2}}{4 b^{2} t}\right]\right\} d \xi \\
& +(\pi k c \rho)^{-1 / 2} \int_{0}^{t} H(t-\tau) \sum_{n=-\infty}^{\infty} \exp \left[-\frac{(x-2 n a)^{2}}{4 b^{2} t}\right] \frac{d \tau}{\tau^{1 / 2}}
\end{aligned}
$$

where $b^{2}=k / c \rho$ is the thermal diffusivity. An equivalent expression in the form of a Fourier series could also be written. The time when melting commences, $t=t_{m}$, will be given by $T_{m}=T\left(0, t_{m}\right)$. If melting stops at any later time $t=t_{1}$, then during the period when $T\left(s\left(t_{1}\right), t\right)<T_{m}$, the temperature will be given by the expression similar to (2.11) but with the origin of distance and time coordinates at $s\left(t_{1}\right)$ and $t_{1}$.

$$
\begin{aligned}
T(x, t) & =\left[4 b^{2} \pi\left(t-t_{1}\right)\right]^{-1 / 2} \int_{s\left(t_{1}\right)}^{a} T\left(\xi, t_{1}\right) \sum_{n=-\infty}^{\infty}\left\{\exp \left[-\frac{\left[x-\xi+2 n\left(a-s\left(t_{1}\right)\right]^{2}\right.}{4 b^{2}\left(t-t_{1}\right)}\right]\right. \\
& \left.+\exp \left[-\frac{\left[x+\xi-2(n+1) a+2 n s\left(t_{1}\right)\right]^{2}}{4 b^{2}\left(t-t_{1}\right)}\right]\right\} d \xi \\
& +(\pi k c \rho)^{-1 / 2} \int_{t_{1}}^{t} H(t-\tau) \sum_{n=-\infty}^{\infty} \exp \left[-\frac{\left[x-2 n a+(2 n-1) s\left(t_{1}\right)\right]^{2}}{4 b^{2}\left(t-t_{1}\right)}\right] \frac{d \tau}{\left(\tau-t_{1}\right)^{1 / 2}}
\end{aligned}
$$

Instead of the heat input rate $H(t)$ being given as a function of time, the problem could also be stated with heat input defined by the usual "radiation" condition as $h(t)\left[T_{o}(t)-T(s(t), t)\right]$, where $T_{\sigma}$ is the temperature of the radiating medium. If $T_{\sigma}$ is large compared with the surface temperature, the two statements will be practically equivalent. In the rest of this paper we take $H$ as constant, which is the same as putting $h$ and $T_{o}$ constant during melting.

3. The semi-infinite solid. We now consider a much more special problem. In many cases occurring in practice the melting will proceed only a relatively small distance into the solid, so that it will be sufficiently accurate to take $a=\infty$, that is, a semiinfinite solid. At the same time we assume that the thermal properties $c$ and $k$, heat input rate $H$, and initial temperature $T_{0}$ are constant with $H>0$. In some applications the variation of these quantities may be important. Even for these cases the results given here are useful as an approximation, and in showing how the progress of the melting depends on the physical quantities involved. By assuming these parameters to be constant we can also give a more detailed treatment which covers the entire range of physically possible values of the parameters.

The condition (2.3) now becomes

$$
\frac{\partial T}{\partial x} \rightarrow 0, \quad x \rightarrow \infty, \quad t>0
$$

which is equivalent to

$$
T \rightarrow T_{0}, \quad x \rightarrow \infty, t>0 .
$$


This equivalence can be seen from

$$
\iint_{D}\left(\frac{\partial T}{\partial x}\right)^{2} d x d t=\int_{C}\left[\frac{T^{2}}{2 b^{2}} d x+T \frac{\partial T}{\partial x} d t\right]
$$

applied to a region $D$ for which $x$ is sufficiently large. Equation (3.3) like (2.7) is a form of Green's theorem for the heat conduction equation, ${ }^{5}$ and can be obtained by applying Gauss's theorem to $\partial\left(T^{2}\right) / \partial t-b^{2} \partial^{2}\left(T^{2}\right) / \partial x^{2}$ where $T$ satisfies Eq. (2.1).

The solution of the problem before melting starts is now, either from (2.11), or directly, ${ }^{6}$

$$
\begin{aligned}
T(x, t) & =T_{0}+2 H\left(\frac{t}{k c \rho}\right)^{1 / 2}\left[\pi^{1 / 2} \exp \left(-\frac{x^{2}}{4 b^{2} t}\right)-\frac{x}{2 b t^{1 / 2}} \operatorname{erfc}\left(\frac{x}{2 b t^{1 / 2}}\right)\right] \\
& =T_{0}+2 H\left(\frac{t}{k c \rho}\right)^{1 / 2} \operatorname{ierfc}\left(\frac{x}{2 b t^{1 / 2}}\right),
\end{aligned}
$$

where ierfe is the notation for the integral of the error function complement introduced by Hartree. ${ }^{7}$

From (3.4) the time when melting starts, $t_{m}$, is given by

$$
t_{m}=\frac{\pi}{4} \frac{k c \rho}{H^{2}}\left(T_{m}-T_{0}\right)=\left(\rho \frac{L}{H} b m\right)^{2} .
$$

Here

$$
m=\frac{\pi^{1 / 2}}{2} \frac{c\left(T_{m}-T_{0}\right)}{L}
$$

is the ratio of the heat content change from $T_{0}$ to $T_{m}$ to the heat of fusion, multiplied by a convenient factor. By the transformations given below the whole problem during melting is reduced to a form in which the only parameter is $m$.

In this semi-infinite case it is possible to eliminate the moving boundary by introducing a new distance variable $x-s(t)$, the distance being measured from the moving face of the melting solid. ${ }^{8}$ Since we now have left to consider only the melting process, we shall measure time from $t_{m}$. We also make the variables dimensionless so as to obtain the equations in the desired simplified form. The new variables are

$$
\begin{gathered}
v=v(z, y)=\frac{T-T_{0}}{\pi^{1 / 2}\left(T_{m}-T_{0}\right)}, \\
z=\frac{x-s(t)}{b t_{m}^{1 / 2}}
\end{gathered}
$$

'E. Coursat, Cours d'analyse mathematique, vol. 3, Gauthier-Villars, Paris, 1915, p. 308.

${ }^{6} \mathrm{R}$. V. Churchill, Modern operational mathematics in engineering, McGraw-Hill, New York, 1944, p. 107.

${ }^{7} \mathrm{D}$. R. Hartree, Properties and applications of the repeated integrals of the error function, Mem. and Proc. Manchester Lit. and Phil. Soc. 80, 85-102 (1935-36).

${ }^{8}$ Since $x-s(t)=\lim _{a \rightarrow \infty} a[1-(a-x) /(a-s(t))]$, this is a special case of the transformation defined by $\xi$ above, not an entirely different transformation for eliminating the moving boundary. 


$$
\begin{gathered}
y=\frac{t}{t_{m}}-1, \\
\sigma=\frac{\rho L}{H} \frac{s}{t_{m}}, \\
\mu=\frac{d \sigma}{d y}=\frac{\rho L}{H} \frac{d s}{d t} .
\end{gathered}
$$

Then the equations for the melting process become

$$
\begin{gathered}
\frac{\partial v}{\partial y}=\frac{\partial^{2} v}{\partial z^{2}}+m \mu(y) \frac{\partial v}{\partial z}, \quad z>0, \quad y>0 \\
v=\operatorname{ierfc}\left(\frac{z}{2}\right), \quad z \geq 0, \quad y=0 \\
v \rightarrow 0, \quad z \rightarrow \infty, \quad y \geq 0 \\
1=-2 \frac{\partial v}{\partial z}+\mu(y), \quad z=0, \quad y>0 \\
v=\pi^{-1 / 2}, \quad z=0, \quad y \geq 0
\end{gathered}
$$

This form for the equations is convenient because there is only one parameter $m$, and it occurs only in the differential equation (3.12); the initial and boundary conditions contain no parameters. This form for the equations, therefore, greatly simplifies the numerical integration which is described later.

Equation (3.15) should be regarded as the definition of the unknown reduced rate of melting, $\mu(y)$. It is possible to eliminate $\mu$ from the equations by substituting its value from (3.15) into (3.12). Equation (3.12) then becomes

$$
\frac{\partial v}{\partial y}=\frac{\partial^{2} v}{\partial z^{2}}+m\left[1+2 \frac{\partial v(0, y)}{\partial z}\right] \frac{\partial v}{\partial z}
$$

which is clearly non-linear.

4. Steady state solution. We first note that there is a steady state solution for the system (3.12) to (3.16); that is, the temperature of the melting, semi-infinite solid approaches a state characterized by the migration inward at a constant velocity of a fixed temperature distribution. It is found from the numerical integration that $\partial v / \partial y \geq 0$. Since $v \leq \pi^{-1 / 2}$ it follows that $\partial v / \partial y \rightarrow 0$ as $y \rightarrow \infty$ and that $v$ tends to a steady state. If we put $\partial v / \partial y=0$, then $\mu$ is constant, say $\mu=\mu_{e}$, the constant steady state value of the reduced melting rate. From (3.12), (3.14), (3.15) and (3.16) this steady state is found to be given by

$$
v \rightarrow v(z)=\pi^{-1 / 2} \exp \left(-m_{\mu_{0}} z\right)
$$

with

$$
\mu_{\circ}=\left(1+2 m \pi^{-1 / 2}\right)^{-1} \text {. }
$$


For the steady state rate of melting in terms of the original variables, this gives

$$
\frac{d s}{d t} \rightarrow V=\frac{H}{\rho\left[L+c\left(T_{m}-T_{0}\right)\right]},
$$

which is also obvious physically. This does not give the steady state value for the thickness melted, but if we use, as a first approximation, a constant rate of melting, then the steady state thickness melted is approximately,

$$
\sigma(y) \cong \mu_{e} y, \quad \text { or } \quad s(t) \cong V\left(t-t_{m}\right),
$$

and the steady state temperature distribution in the original variables becomes

$$
T \cong T_{0}+\left(T_{m}-T_{0}\right) \exp \left\{-V b^{-2}\left[x-V\left(t-t_{m}\right)\right]\right\} \text {. }
$$

Actually the melting rate is initially less than its steady state value, so that (4.4) and (4.5) give values for the thickness melted and the temperature which are too large. We can obtain the exact steady state value as follows.

Equation (2.8) written in terms of the reduced variables is

$$
\sigma(y)=\mu_{e}\left(y+1-2 \int_{0}^{\infty} v(z, y) d z\right) .
$$

Then by use of (4.1), the steady state value of $\sigma$ is

$$
\sigma(y) \rightarrow \sigma_{e}(y)=\mu_{e}(y+1)-2 / \pi^{1 / 2} m,
$$

or correspondingly

$$
s(t) \rightarrow V t-(k / H)\left(T_{m}-T_{0}\right),
$$

and the exact steady state value for $T$ is

$$
T \rightarrow T_{0}+\left(T_{m}-T_{0}\right) \exp \left\{-V b^{-2}\left[x-V t+k\left(T_{m}-T_{0}\right) / H\right]\right\} .
$$

Since $v(z, y)$ increases with $y$, it can be seen from (4.6) that the steady state value gives a lower bound for the thickness melted. Also since

$$
\int_{0}^{\infty} v(z, 0) d z=\int_{0}^{\infty} \operatorname{ierfc}(z / 2) d z=1 / 2,
$$

(4.4) gives an upper bound; that is,

$$
V\left(t-t_{m}\right) \geq s(t)>V t-(k / H)\left(T_{m}-T_{0}\right) .
$$

The difference between these two bounds is

$$
\frac{k\left(T_{m}-T_{0}\right)}{H}\left[1-\frac{\pi}{4}\left(1+\frac{L}{c\left(T_{m}-T_{0}\right.}\right)^{-1}\right] .
$$

In the applications, this quantity is often small; then the steady state value is a good approximation of $s(t)$ for all $t>t_{m}$.

By taking derivatives of (4.6) we find that the melting rate is always less than its steady state value:

$$
\mu(y)<\mu_{e}, \quad \text { or } \frac{d s}{d t}<V .
$$


Physically, this is obvious since the heat content of the solid is increasing during the melting.

5. The case $m=0$. The parameter $m$ in Eq. (3.12) can have any positive value. The value $m=0$ does not correspond to a physically realizable situation but is a limit which is approached when the latent heat $L$ becomes large. In this case, the system (3.12) to (3.16) can be solved in terms of tabulated functions. This solution is of value since it is one limit of the set of solutions for $0<m<\infty$. Also the solution for $m=0$ is an approximation to that for any finite $m$ when $y$ is sufficiently small, because $\mu(0)=0$.

For $m=0$ the equations are linear. Equation (3.10) does not enter into the solution but is merely an equation for determining $\mu(y)$ from the solution. The solution for $m=0$ can be written as

$\dot{v_{0}}(z, y)=\pi^{-1 / 2} \operatorname{erfc}(z / 2 y)$

$$
+(4 \pi y)^{-1 / 2} \int_{0}^{\infty} \operatorname{ierfc}\left(\frac{z}{2}\right)\left\{\exp \left[-\frac{(z-\zeta)^{2}}{4 y}\right]-\exp \left[-\frac{(z+\zeta)^{2}}{4 y}\right]\right\} d \zeta .
$$

The indicated integrations are rather tedious; hence we omit the details and give only the final result.

$$
\begin{aligned}
v_{0}(z, y)=\pi^{-1 / 2} & \operatorname{erfc}(z / 2 y)-(z / \pi) \arctan \left(y^{-1 / 2}\right) \\
+ & {[(y+1) / \pi]^{1 / 2} \exp \left[-z^{2} / 4(y+1)\right] \operatorname{erf}\left\{z / 2[y(y+1)]^{1 / 2}\right\} } \\
+ & 2 z W\left(z /[2(y+1)]^{1 / 2}, y^{-1 / 2}\right)
\end{aligned}
$$

where

$$
W(\alpha, \beta)=(2 \pi)^{-1} \int_{0}^{\alpha} \int_{0}^{\beta x} \exp \left[-\left(x^{2}+y^{2}\right) / 2\right] d y d x
$$

is a tabulated function. ${ }^{9}$

This gives for the reduced rate of melting

and hence

$$
\mu_{0}(y)=(2 / \pi) \arctan \left(y^{1 / 2}\right),
$$

$$
\sigma_{0}(y)=(2 / \pi)\left[(y+1) \arctan \left(y^{1 / 2}\right)-y^{1 / 2}\right],
$$

which as stated above are approximations to $\mu(y)$ and $\sigma(y)$ for finite $m$ when $y$ is small.

Since $v_{0}(z, y)$ can be used to start the numerical integration for $0<m<\infty$, it is useful to have simpler expressions than (5.2). For $y>0, v_{0}(z, y)$ is analytic, and we can expand it in a power series in $z$. By use of $\partial v_{0} / \partial y=\partial^{2} v_{0} / \partial z^{2}$ and $\partial v_{0} / \partial z=$ $-\pi^{-1}$ arc tan $\left(y^{-1 / 2}\right)$ at $z=0$, this expansion is

$$
\begin{aligned}
v_{1}(z, y) & =\pi^{-1 / 2}-\pi^{-1} \sum_{n=0}^{\infty} \frac{d^{n}}{d y^{n}}\left[\arctan \left(y^{-1 / 2}\right)\right] \frac{z^{2 n+1}}{(2 n+1) !} \\
& =\pi^{-1 / 2}-\frac{z}{\pi}\left[\arctan \left(y^{-1 / 2}\right)-\frac{z^{2}}{12 y^{1 / 2}(y+1)}+\cdots\right], \quad y>0 .
\end{aligned}
$$

${ }^{9} \mathrm{C}$. Nicholson, The probability integral for two variables, Biometrika 33, 59-72 (1943). Also, J. B. Rosser, Theory and application of $\int_{0}^{t} \exp \left(-x^{2}\right) d x$ and $\int_{0}^{z} \exp \left(-p^{2} y^{2}\right) d y \int_{0}^{y} \exp \left(-x^{2}\right) d x$, Mapleton House, Brooklyn, 1948. 
Also for $z / y^{1 / 2}$ large

$$
\begin{aligned}
W\left(z /[2(y+1)]^{1 / 2}, y^{-1 / 2}\right) \cong \frac{1}{4} \operatorname{erf} & {\left[z / 2(y+1)^{1 / 2}\right] } \\
& -\frac{1}{2 \pi} \arctan \left(y^{1 / 2}\right),
\end{aligned}
$$

so that we get

$$
v_{0}(z, y) \cong(y+1)^{1 / 2} \text { ierfc }\left[z / 2(y+1)^{1 / 2}\right] .
$$

This relation shows that when melting begins, the temperature in the interior is nearly the same as it would be without melting.

6. The case $m=\infty$. The case $L=0$, which makes $m=\infty$, is of interest for its physical applications and also because it gives the other limit to the range of $m$. In fact for steel, with $T_{0}$ as room temperature, $m=27$, which is "practically infinite" in the sense that the temperature and melting rates will be very close to those for $m=\infty$.

For $L=0$, the definitions of $\sigma$ and $\mu$ in (3.10) and (3.11) must be modified. We introduce the modified quantities

$$
\begin{gathered}
\omega=m \sigma=\frac{2}{\pi^{1 / 2}} \frac{H s}{k\left(T_{m}-T_{0}\right)}, \\
\lambda=m \mu=\frac{d \omega}{d y}=\frac{\pi^{1 / 2}}{2} \frac{c \rho}{H}\left(T_{m}-T_{0}\right) \frac{d s}{d t},
\end{gathered}
$$

which are independent of $L$. In the system (3.12) to (3.16), Eq. (3.12) is now

$$
\frac{\partial v}{\partial y}=\frac{\partial^{2} v}{\partial z^{2}}+\lambda(y) \frac{\partial v}{\partial z}, \quad z>0, \quad y>0,
$$

and (3.15) which came from (2.4) is now

$$
1=-2 \frac{\partial v}{\partial z}, \quad z=0, \quad y>0 .
$$

There is now no equation defining $\lambda$, similar to (3.15) for $\mu$. However, from (3.16), $\partial v / \partial y=0$ at $z=0$, so that if the derivatives are continuous at $z=0, y>0$, Eq. (6.3) gives

$$
\frac{\partial^{2} v}{\partial z^{2}}+\lambda(y) \frac{\partial v}{\partial z}=0, \quad z=0, \quad y>0
$$

or by use of (6.4)

$$
\lambda(y)=2 \frac{\partial^{2} v}{\partial z^{2}}, \quad z=0, \quad y>0,
$$

which now takes the place of (3.15). The modified system of equations is then (6.3), (3.13), (3.14), (6.5), (3.16).

For numerical integration this system is unsatisfactory because the determination of $\lambda$ from (6.5) requires the calculation of a second derivative. Since this is not easily done with accuracy, we consider instead a derived system for $\partial v / \partial z$. 
If we put

$$
u(z, y)=-2 \frac{\partial v}{\partial z}, \quad v=\pi^{-1 / 2}-\frac{1}{2} \int_{0}^{z} u(\zeta, y) d \zeta,
$$

then $u(z, y)$ will satisfy

$$
\begin{gathered}
\frac{\partial u}{\partial y}=\frac{\partial^{2} u}{\partial z^{2}}+\lambda(y) \frac{\partial u}{\partial z}, \quad z>0, \quad y>0, \\
u=\operatorname{erfc}(z / 2), \quad z \geq 0, \quad y=0, \\
u \rightarrow 0, \quad z \rightarrow \infty, \quad y \geq 0, \\
\lambda(y)=-\frac{\partial u}{\partial z}, \quad z=0, \quad y>0, \\
u=1, \quad z=0, \quad y \geq 0,
\end{gathered}
$$

and it is easy to verify that if $u$ satisfies (6.7) to (6.11) then $v$ will satisfy (6.3), (3.13), (3.14), (6.5), (3.16).

An important difference between the present case, $L=0$, and $L>0$, is that for $L=0$ the melting rate is not zero at the start of melting; instead we now have

corresponding to

$$
\lambda(0)=\pi^{-1 / 2}
$$

$$
\frac{d s\left(t_{m}\right)}{d t}=\frac{2 H}{\pi c \rho\left(T_{m}-T_{0}\right)} .
$$

For $L>0, d s\left(t_{m}\right) / d t=0$ was necessary for continuity of $\partial T / \partial x$ in $t$ at $t=t_{m}$, but with $L=0$ this condition is not necessary, as can be seen from (2.4).

The steady state solution is now instead of (4.1),

$$
v_{0}=\pi^{-1 / 2} \exp \left(-\pi^{1 / 2} z / 2\right),
$$

and the steady state values of $\lambda$ and $\omega$ are

$$
\lambda_{c}=\pi^{1 / 2} / 2, \quad \text { and } \quad \omega_{c}=\lambda_{c}(y+1-4 / \pi) .
$$

The steady state equations in terms of the original variables are unchanged except that now $L=0$.

7. Numerical integration. For $m>0$, the solution was obtained by numerical integration using finite differences. We describe the method used, but do not go into a discussion of methods for numerical solution of the heat conduction equation and their convergence and stability. These questions have been discussed by several writers, ${ }^{10}$ although much remains to be done, especially for non-linear problems.

${ }^{10}$ R. Courant, K. Friedrichs and H. Lewy, Über die Partiellen Differenzenglieichungen der Mathematischen Physik, Math. Ann. 100, 32-74 (1928); N. R. Eyres, D. R. Hartree, et al., The calculation of variable heat flow in solids, Proc. Roy. Soc. Lond. (A) 240, 1-57 (1946); C. M. Fowler, Analysis of numerical solutions of transient heat-flow problems, Q. Appl. Math. 3, 361-376 (1946); J. Crank and P. Nicolson, $A$ practical method for numerical evaluation of solutions of partial differential equations of the heat-conduction type, Proc. Camb. Phil. Soc. 43, 50-67 (1947). Unpublished methods proposed by J. von Neumann. 
We use $\Delta z$ and $\Delta y$ for the intervals in $z$ and $y$, and write $v(i, j)$ for $v(z, y)=v(i \Delta z, j \Delta y)$ and $\mu_{i}$ for $\mu(j \Delta y)$. The time derivative is approximated by a forward difference ratio and the space derivatives by central difference ratios,

$$
\begin{gathered}
\frac{\partial v(i, j)}{\partial y} \cong(\Delta y)^{-1}[v(i, j+1)-v(i, j)], \\
\frac{\partial v(i, j)}{\partial z} \cong(2 \Delta z)^{-1}[v(i+1, j)-v(i-1, j)], \\
\frac{\partial^{2} v(i, j)}{\partial z^{2}} \cong(\Delta z)^{-2}[v(i+1, j)-2 v(i, j)+v(i-1, j)],
\end{gathered}
$$

so that Eq. (3.12) is approximated by the difference equation

$$
\begin{aligned}
v(i, j+1)-v(i, j)=\frac{\Delta y}{\Delta z^{2}} & \{v(i+1, j)-2 v(i, j) \\
& \left.+v(i-1, j)+m \mu_{i} \frac{\Delta z}{2}[v(i+1, j)-v(i-1, j)]\right\} .
\end{aligned}
$$

It is known that the solution of the difference approximation to the heat conduction equation will not be stable unless the ratio $\Delta y / \Delta z^{2} \leq 1 / 2$. Stability means that errors which occur in the process of solution, such as rounding-off errors, are damped out instead of being magnified. We then take $\Delta y / \Delta z^{2}=1 / 2$, which simplifies (7.4) by removing the term in $v(i, j)$, so that the difference equation becomes

$v(i, j+1)=\frac{1}{2}\left(1+m \mu_{i} \Delta z / 2\right) v(i+1, j)$

$$
+\frac{1}{2}\left(1-m \mu_{i} \Delta z / 2\right) v(i-1, j) .
$$

To compute $\mu$, a third order formula for $\partial v / \partial z$ at $z=0$ was used. Then from (3.15) and (3.16),

$$
\mu_{j}=1+(\Delta z)^{-1}\left[-11 / 3 \pi^{1 / 2}+6 v(1, j)-3 v(2, j)+\frac{2}{3} v(3, j)\right] .
$$

The computation proceeded along "lines," the values for fixed $j$ and all $i$ being computed before going on to $j+1$. The initial values are given by (3.13), or we can start with $j=1$ using the solution for $m=0$, Eq. (5.2). Having $v(i, j),(7.6)$ is used to find $\mu_{i}$ and then (7.5) is used to compute $v(i, j+1)$. The computation was carried to values of $i$ large enough to make $v(i, j)<5 \times 10^{-7}$.

The calculations were carried out partly on the Bell Relay Computer and mainly 'on the I B M Relay Calculator ${ }^{11}$ at the Ballistic Research Laboratories for $m=0.2$, $1,2,5,10$ and $\infty$. The modifications necessary for $m=\infty$ are clear from Sec. 6 .

In the applications of the results a very high accuracy in $\mu$ and $\sigma$ is not needed. The calculations were carried out so as to give $\mu$ (which is the quantity with the largest

${ }^{11}$ These machines are described in F. L. Alt, A Bell Telephone Laboratories computing machine, Math. Tables and Aids Computation 3, 1-13, 69-84 (1948), and W. J. Eckert, The I B M pluggable sequence relay calculator, Math. Tables and Aids Computation 3, 149-161 (1948). 
error) generally correct to three significant figures, although the third figure was sometimes in doubt. The accuracy was checked in various ways, especially by using Eq. (4.6).

Since $\mu$ and $v$ change rapidly at first, it was necessary to start the computation with small values of $\Delta z$ and $\Delta y$. When the rate of change decreased, the interval size had to be increased to keep the computation from becoming too lengthy. As the steady state solution was approached, it would have been desirable to use relatively large intervals of reduced time $\Delta y$, but the limitation $\Delta y / \Delta z^{2} \leq 1 / 2$ would then have required $\Delta z$ to be so large as to introduce serious errors. The limitation on the relative size of the time and the space interval could have been avoided by using a more complicated difference approximation.

To extend the values of $\mu$ to the steady state value, a semi-empirical method was used, as follows. If in Eq. (3.12), $m \mu(y)$ is replaced by a constant $r$, then the system (3.12), (3.13), (3.14) and (3.16) can be solved by using the substitution $v=w(z, y) \exp [-r z / 2-$ $\left.r^{2} y / 4\right]$, to eliminate the term $r \partial v / \partial z$. The solution is then,

$$
\begin{gathered}
v(z, y)=\frac{\exp \left[-r z / 2-r^{2} y / 4\right]}{(4 \pi y)^{1 / 2}} \int_{0}^{\infty} \operatorname{ierfc}(\zeta / 2) e^{r / 2}\left\{\exp \left[-\frac{(z-\zeta)^{2}}{4 y}\right]\right. \\
\left.-\exp \left[-\frac{(z+\zeta)^{2}}{4 y}\right]\right\} d \zeta \\
+\frac{z}{2 \pi} \int_{0}^{y} \exp \left[\frac{r^{2} \eta}{4}-\frac{z^{2}}{4(y-\eta)}\right] \frac{d \eta}{(y-\eta)^{3 / 2}} .
\end{gathered}
$$

The second term $v_{2}(z, y)$ can be evaluated as

$$
v_{2}(z, y)=(4 \pi)^{-1 / 2}\left[\operatorname{erfc}\left(\frac{z+r y}{2 y^{1 / 2}}\right)+e^{-r z} \operatorname{erfc}\left(\frac{z-r y}{2 y^{1 / 2}}\right)\right] .
$$

As $y \rightarrow \infty$, the first term in (7.7) approaches zero, while $v_{2}(z, y) \rightarrow \pi^{-1 / 2} e^{-r z}$, the steady state solution. We then use $\partial v_{2}(0, y) / \partial z$ to indicate the dependence of $u$ on $y$, for $y$ large enough so that $\mu$ is almost equal to $\mu_{e}$. From (7.8)

$$
\frac{\partial v_{2}(0, y)}{\partial z}=-\pi^{-1 / 2}\left[r+y^{-1 / 2} \operatorname{ierfc}\left(r y^{1 / 2} / 2\right)\right],
$$

Putting $r=m \mu$, and using (3.15) we have

$$
\begin{aligned}
\mu & \cong \mu_{e}\left[1-2(\pi y)^{-1 / 2} \operatorname{ierfc}\left(m \mu y^{1 / 2} / 2\right)\right] \\
& \cong \mu_{e}\left[1-2(\pi y)^{-1 / 2} \operatorname{ierfc}\left(m \mu_{\odot} y^{1 / 2} / 2\right)\right]
\end{aligned}
$$

for large $y$.

It was found that for $y$ large the computed values of $\mu$ varied with $y$ in this way, except that the second term in (7.10), which is a small correction term, had to be multiplied by a constant depending on $m$. Thus $\mu$ could be extended to arbitrarily large $y$ with an error which is within the desired limits, because the extension was started for $\mu$ within a few percent of $\mu_{e}$. 
Figures 1 and 2 show the values of the melting rate plotted against time for $m=0$, $0.2,1,2,5,10$ and $\infty$. In Fig. 1 it can be seen that for all $m$, the values of $\mu$ approximate

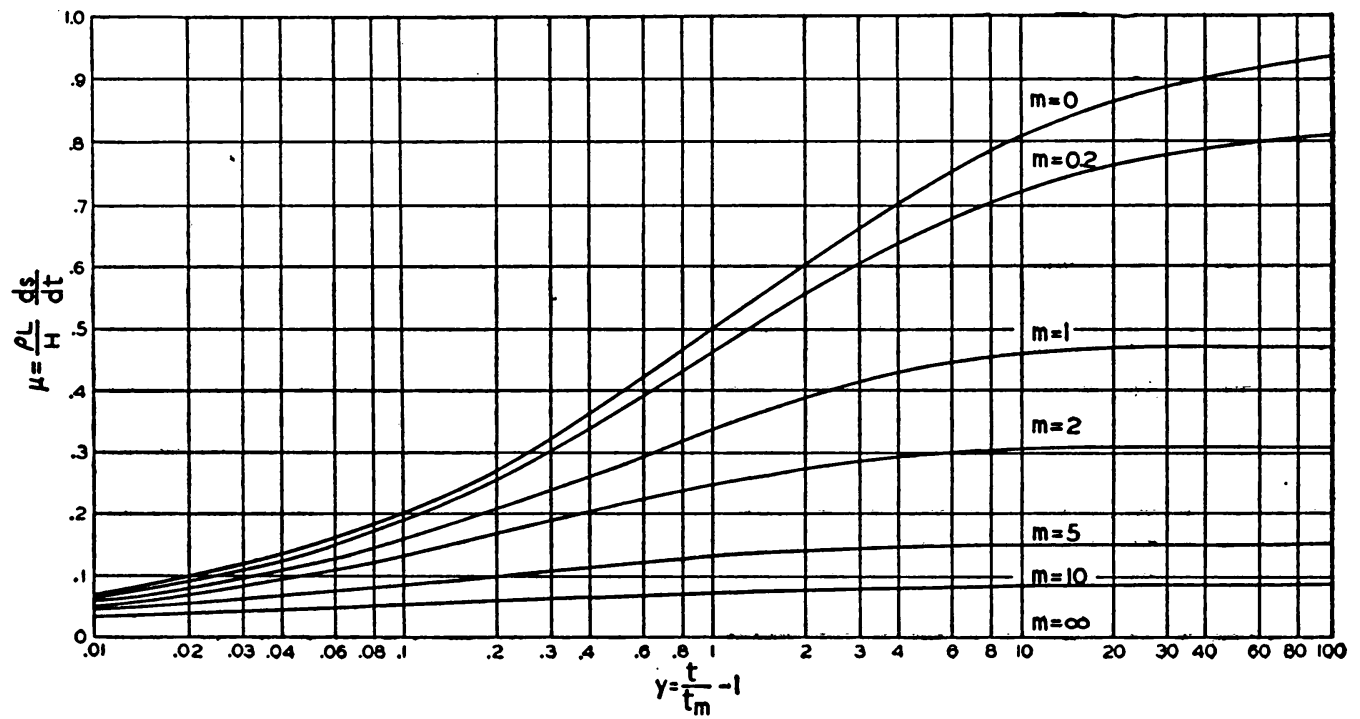

Frg. 1. Rate of melting of the semi-infinite solid for various values of $m$ : $m=\pi^{1 / 2} c\left(T_{m}-T_{0}\right) / 2 L, d s / d t=$ rate of melting, $\rho=$ density, $L=$ latent heat, $H=$ heat input rate per unit area, $t=$ time, $t_{m}=$ time when melting begins.

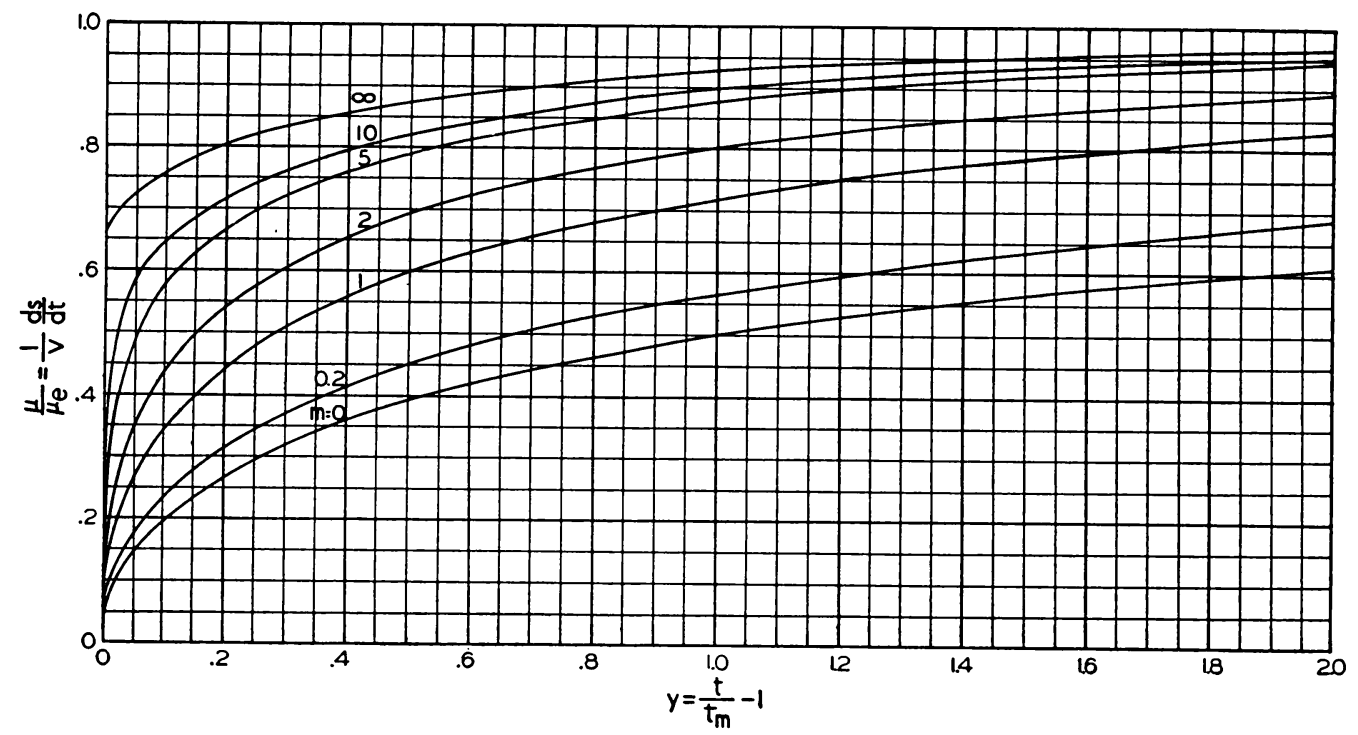

FIG. 2. Rate of melting as fraction of the steady state rate: $\mathrm{V}=H / \rho\left[L+c\left(T_{m}-T_{0}\right)\right]$, steady state rate of melting.

those for $m=0$ for small values of $y$, and that $\mu$ approaches its steady state value more rapidly the larger the value of $m$. In Fig. 2 the ordinate is $\mu / \mu_{e}$ (for $m=\infty$, it is $\lambda / \lambda_{\bullet}$ ) 
so that all the curves approach unity as an asymptote. In this figure it can be seen that as $m$ becomes large $\mu / \mu_{o}$ near $y=0$ approaches the value for $m=\infty$, although the value of $\mu / \mu_{\mathrm{e}}$ at $y=0$ is quite different from that of $m=\infty$. Figure 3 shows the thickness melted; the asymptotic values here are $\sigma / \mu_{e} \rightarrow y+1-2 /\left(\pi^{1 / 2} m \mu_{e}\right)$ for $m>0$, and $y+1-4 y^{1 / 2} / \pi$ for $m=0$.

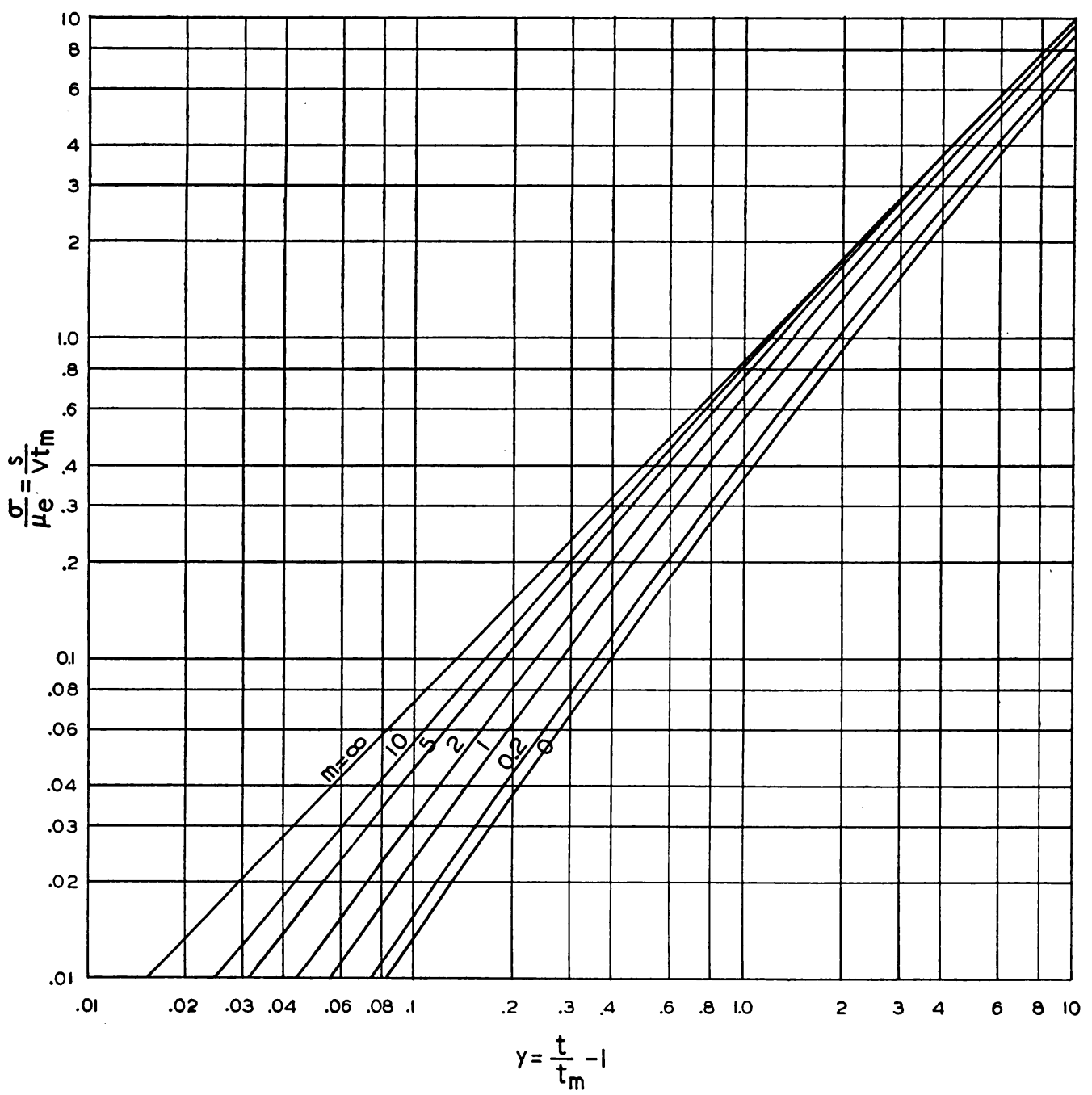

FIG. 3. Thickness melted, $s$.

8. Acknowledgments. The writer gratefully acknowledges the assistance of the following members of the Ballistic Research Laboratories: Bruce L. Hicks, who collaborated at the beginning of the work on this problem and contributed several points incorporated above; E. E. Kolin, who performed necessary hand calculations; J. H. Levin and J. O. Harrison, who set up and supervised the calculations on the I B M and Bell Relay Calculators, respectively. 